\title{
Polymer materials with controlled degradation time
}

\author{
Ewelina Niedzielska ${ }^{1, *}$, Anna Masek $^{1}$ \\ ${ }^{1}$ Lodz University of Technology, Institute of Polymer and Dye Technology, Lodz, Poland
}

\begin{abstract}
The aim of this work was carry out accelarated process of ageing for cyclic olefin copolymer ethylene - norbornene (Topas). The Topas cyclic olefin copolymer (COC) family characterize high transparency, excellent mechanical properties and low water permeability. The influence of external factors such as UV radiation, elevated temperature, oxygen effect and humidity causing degradation of polymer macroparticles was also investigated. The properties of the polymer before and after weathering and UV radiation were also compared. Degradability was examinated by measuring color change, FTIR spectrum analysis and determination of the ageing factor $k$. The tensile strength, elongation at break and hardness of composites by Shore A method were measured. The synergistic effect of temperature, humidity and $\mathrm{UV}$ radiation reduces the mechanical properties of the samples tested, while the interaction of only UV radiation on the samples causes a significant change color.
\end{abstract}

\section{Introduction}

Cyclic olefin copolymer with the trade name TOPAS COC (Cyclic Olefin Copolymer) is obtained by copolymerizing nonbornene with ethylene using metallocene catalysts. Among the polyolefines it is distinguished by an amorphous structure thanks to which it is transparent. Its properties can be varied in a wide range during polymerization. It is its unusual features such as low density, very low moisture permeability, resistance to hydrolysis and polar substances have made the material a success. It is also characterized by low birefringence of light, high stiffness and thermal resistance (up to $170^{\circ} \mathrm{C}$ ). It can be an electrical isolation. An additional advantage is that the material described is environmentally friendly and can be easily disposed of. During combustion, it releases only water and carbon dioxide. Through to biocompatibility and physiological indifference, it is readily used in medicine and in the packaging industry (Topas from Ticona has been approved by the American Food and Drug Administration (FDA) for contact with all types of food). Topas has been used as blister foils, ampoules, and vials in the pharmaceutical industry; food films; optical goods, e.g. lenses, sensors; metallized foils for capacitors; and even as injection

\footnotetext{
* Corresponding author: ewelina.niedzielska@edu.p.lodz.pl
} 
molded products reinforced with glass fiber [1-3].We examinated the properties of copolymer etyhlen - norbornene after accelerated process of ageing - weathering and UV radiation and we explored degree of degradation of polymer matrix.

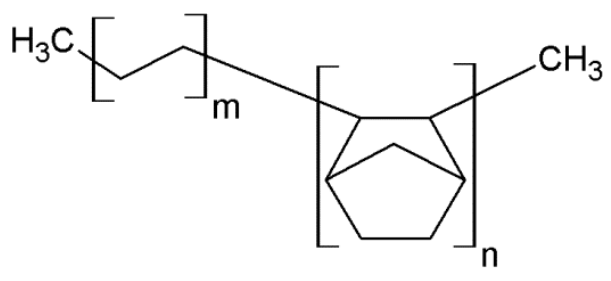

Fig. 1. Structural formula copolymer ethylen - norbornen (Topas)

\section{Materials and Methods}

\subsection{Formation samples}

In our work we used thermoplastic polyolefin elastomer Topas Elastomer E-140 (supplied by Topas Advanced Polymers). The samples were made in two steps. The first is to make polymer blends in a Brabender micromixer, preheated to $110^{\circ} \mathrm{C}$. Then, in order to prepare the tiles for further testing, the polymer was pressed in hydraulic presses at a temperature of $120^{\circ} \mathrm{C}$ for 10 minutes. After this time, polymer tiles with the desired dimensions were obtained.

\subsection{Weathering}

We placed the composite tiles in the "Atlas Weather Ometer Ci 4000" apparatus. A single study lasted 360 minutes and consisted of two alternating segments: daily (duration: 240 minutes, humidity: 50\%, radiation intensity: $0.4 \mathrm{~W} / \mathrm{m}^{2}$ ) and night time (duration: 120 minutes, humidity: $60 \%$, no radiation). The ageing time was up to $1120 \mathrm{~h}$.

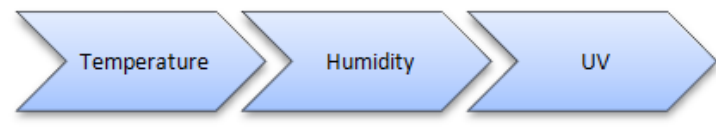

\section{WEATHERING}

Fig. 2. Factors of weathering.

\subsection{UV ageing}

We put the prepared composite tiles in special metal folders and these were installed in the Atlas $2000 \mathrm{UV}$ device. A single measurement lasted 360 minutes and consisted of two alternating segments: daily (duration: 240 minutes, temperature: $60^{\circ} \mathrm{C}$, radiation intensity: $0.7 \mathrm{~W} / \mathrm{m}^{2}$ ) and night time (duration: 120 minutes, temperature: $50^{\circ} \mathrm{C}$, no UV radiation). The ageing time was up to $1120 \mathrm{~h}$. 


\subsection{Mechanical properties}

We carried out the tests according to the standard: ISO PN-82 / C-04205 (PN-ISO $2781+$ AC: 1996) using the "Zwick" testing machine (model 143) for 4 mm wide samples, about $1 \mathrm{~mm}$ thick, at a stretching rate of $500 \mathrm{~mm} / \mathrm{min}$ and initial force $0,1 \mathrm{~N}$. For each specimen, we determined the values: tensile strength TS and the relative elongation at break EB. Polymer hardness measurements were made using a Zwick durometer according to ISO 7619-1: 2010, using the Shore D method, using an indenter according to PN-93 / C-04206.

\subsection{Color change}

The color change of the samples was measured in accordance with PN-EN ISO 105 - J01, using a spectrometer that uses the $\mathrm{dE} *$ ab color model CIE LAB to determine the color difference.

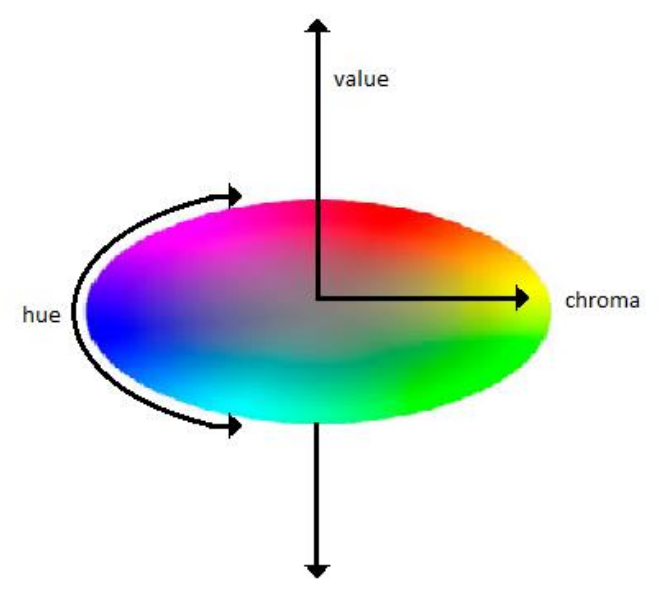

Fig. 3. Model CIE-LAB showed 3 types of Munsell colors [4].

\subsection{ATR-FTIR spectroscopy}

Infrared spectra were measured using a FTIR Nicolet 6700 spectrophotometer and OMNIC 3.2 software (Thermo Scientific Products: Riviera Beach, FL, USA). The analyses were carried out at room temperature and ambient humidity. All the spectra were acquired between 4000 and $700 \mathrm{~cm}^{-1}$ with a spectral resolution of $8 \mathrm{~cm}^{-1}$ and 128 scans in order to exploit the instrumental built-up noise reduction algorithm. The spectra were collected in absorbance mode. Spectrum was collected from an average of 32 scans. 


\section{Discuss}

\subsection{Mechanical of properties}

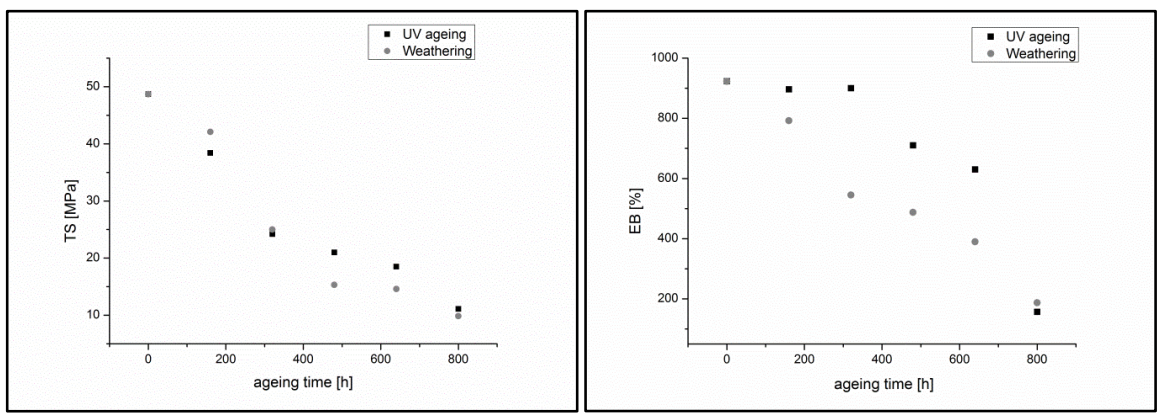

Fig. 4. Tensile strength TS (1) and Elongation at break EB (2) of ethylene-norbornene samples after $800 \mathrm{~h}$ of UV-ageing and weathering.

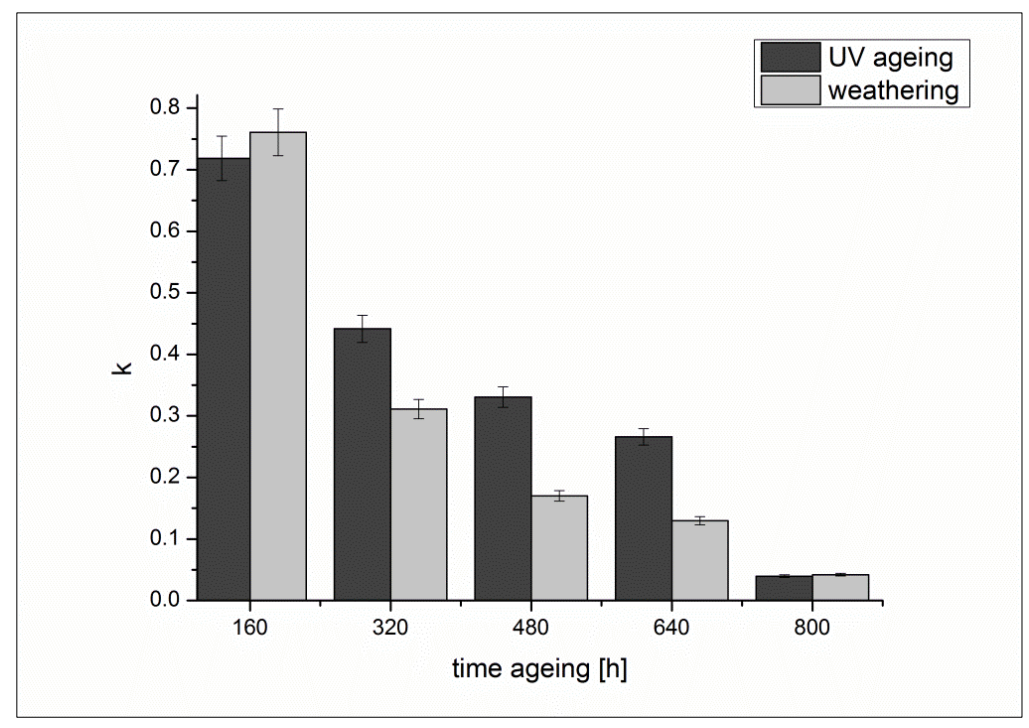

Fig. 5. Ageing coefficient $\mathrm{K}$ for Topas samples after $800 \mathrm{~h}$ of UV-ageing and weathering.

In order to show the degradation progress using formula (1), we determined the ageing factors.

$$
k=\frac{(T S \cdot E B)_{\text {after ageing }}}{(T S \cdot E B)_{\text {before ageing }}}
$$

The value of coefficient $\mathrm{k}$ informs about the degree of degradation of the polymer matrix. When $\mathrm{k} \rightarrow 0$ material degraded, while $\mathrm{k} \rightarrow 1$ material stay stable. 


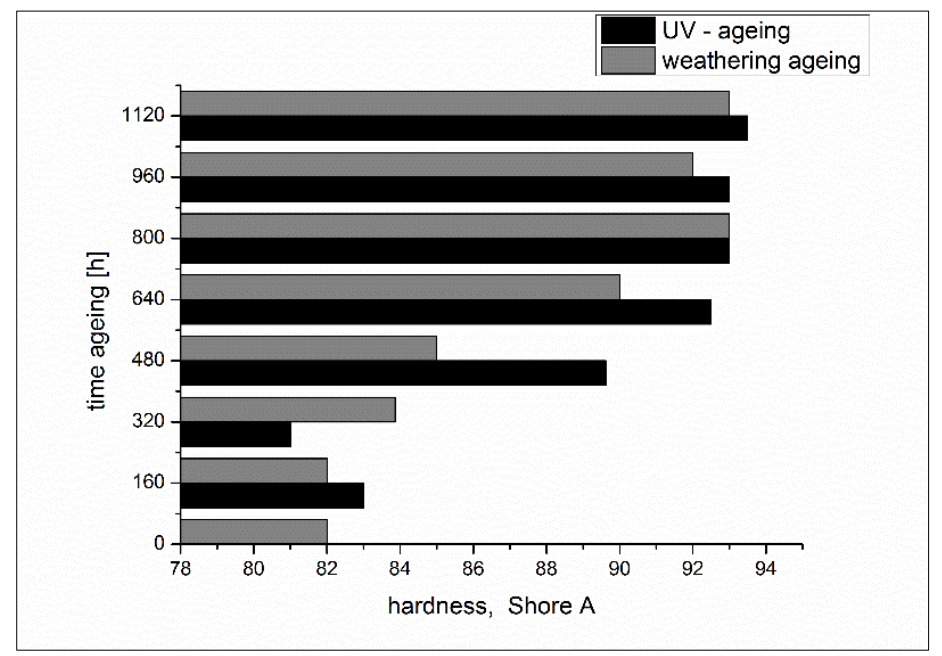

Fig. 6. Shore hardness of Topas samples after $1120 \mathrm{~h}$ of UV-ageing and weathering.

As can be seen in Fig. 3, the standard sample of the ethylene-norbornene copolymer characterize a high tensile strength $(49 \mathrm{MPa})$. After each ageing cycle $(160 \mathrm{~h})$, both weathering and UV light ageing, the mechanical properties (TS, EB) of the polymer are reduced linearly. However, in the case of hardness measurements of composites, along with the ageing time, the determined values are increased, which confirms the prediction that Topas samples after ageing become rigid and hard. Coefficient $\mathrm{k}$ picture change of energy deformation after ageing. We can observed for samples after UV ageing larger decrease coefficient $\mathrm{k}$ than for weathering.

\subsection{Color change}

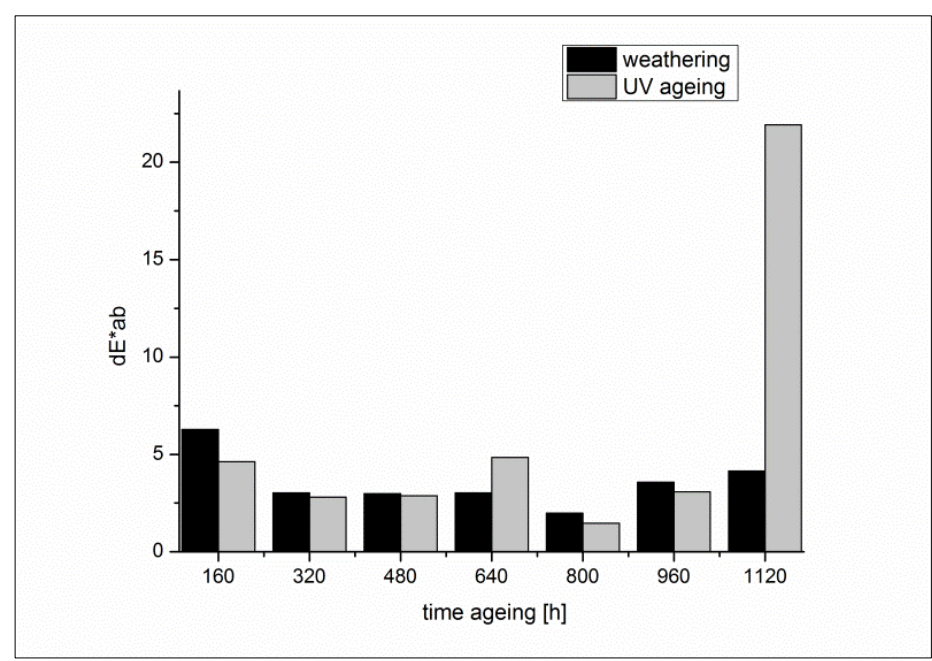

Fig. 7. Coefficient $\mathrm{dE}^{*}$ ab for Topas samples after $1120 \mathrm{~h}$ of UV-ageing and weathering. 


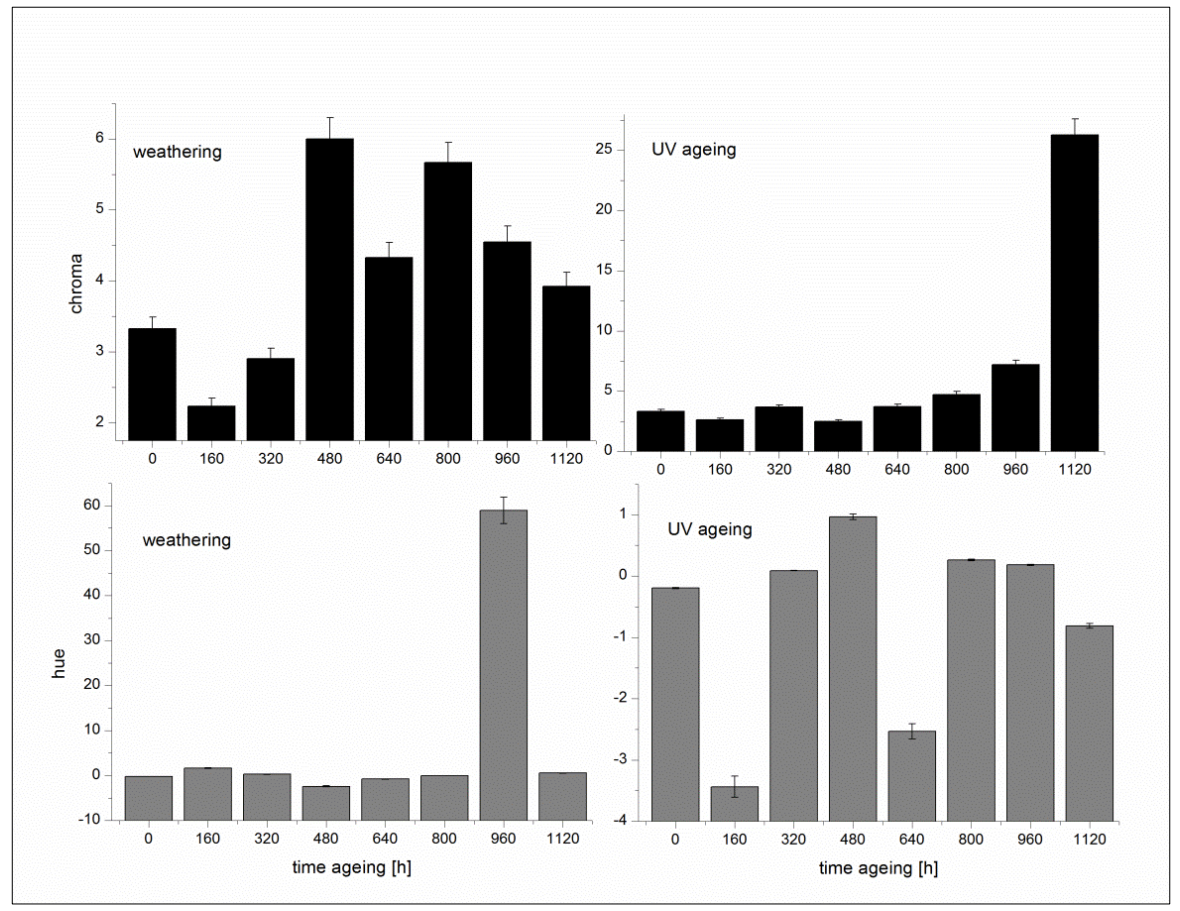

Fig. 8. Hue and chroma of Topas samples after $1120 \mathrm{~h}$ of UV-ageing and weathering.

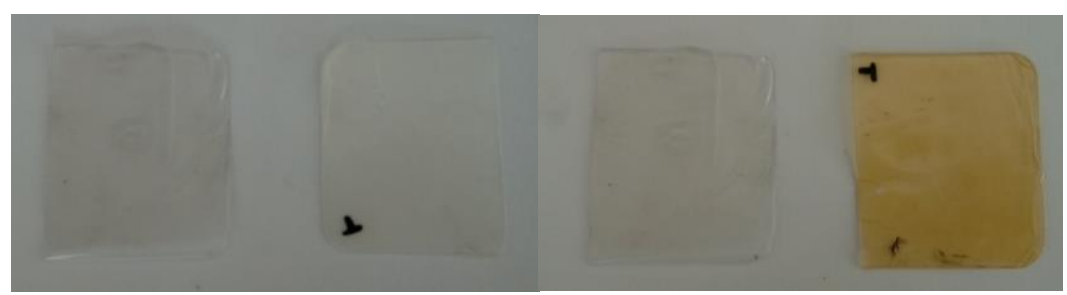

Fig. 9. Reference sample TOPAS and sample after $1120 \mathrm{~h}$ weatherng ageing (1) and reference sample TOPAS (1) and sample after $1120 \mathrm{~h}$ UV ageing (2).

The largest values of $\mathrm{dE}^{*} \mathrm{ab}$ and chroma are characterized by elastomer samples after 1120 hours of UV ageing, which allows us to state that the material has significantly changed color. This is confirmed by the pictures showed a colorless reference sample and a yellow sample after UV ageing (Fig.8). However, there are slight changes in the color of samples after weathering ageing.

\subsection{FTIR of spectrum}

In order to compare the degree of degradation of Topas samples before and during ageing, we performed an FTIR infrared spectrometry test. Absorption bands indicating the carbonyl group (1700-1750 $\mathrm{cm}^{-1}$ ) appear already after 320 hours of ageing and successively increase their intensity along with the ageing time, which can be seen in the attached spectrum (Fig.9.). 


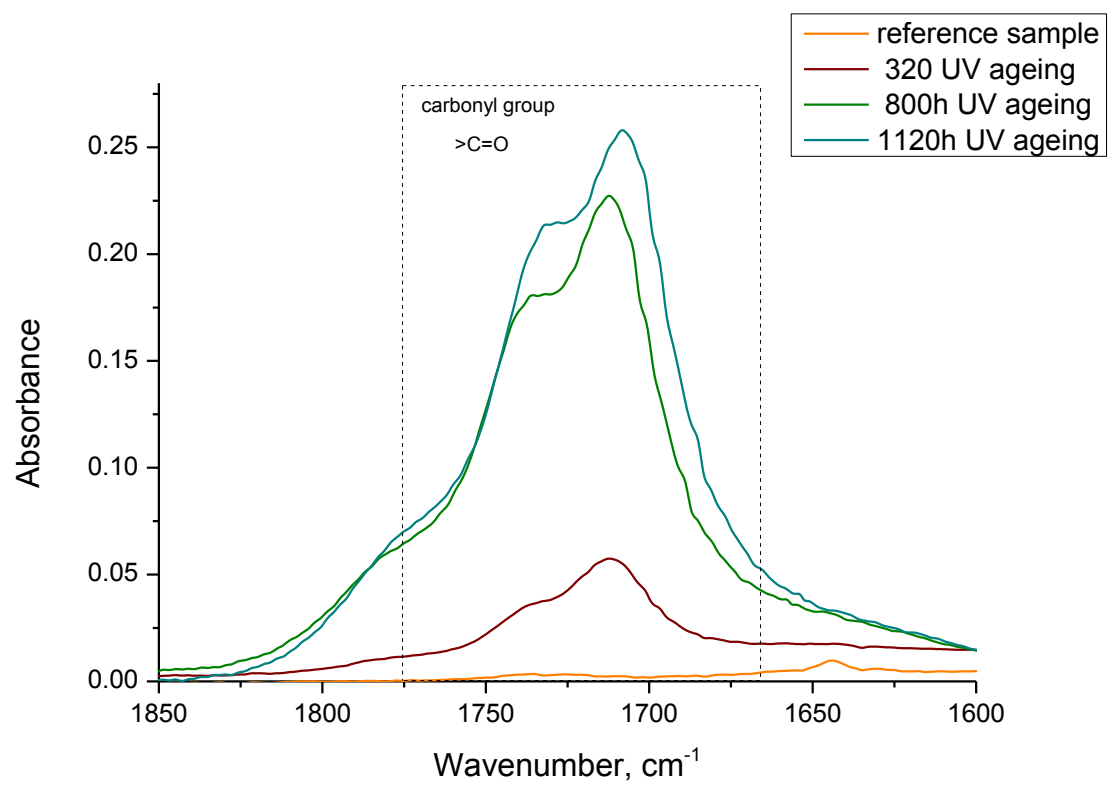

Fig. 10. FTIR spectrum for Topas samples after $320 \mathrm{~h}, 800 \mathrm{~h}$ and $1120 \mathrm{~h}$ of UV-ageing and weathering.

\section{Conclusions}

We examinated impact of ageing on structure of cyclic olefin copolymer Topas. The obtained results show a linear reduction in mechanical properties after ageing. Value of tensile strength TS after UV or weathering ageing decreases by $80 \%$. Ageing coefficient $\mathrm{k}$ is very important parameter for term of polymer degradation. In this case we can observed significant decrease energy deformation of polymer. The value of the $\mathrm{dE}^{*}$ ab coefficient allows to determine influence ageing on the color of the polymer. Analysis of FTIR spectra showed significant changes in intensity for the wavelength range $1700-170 \mathrm{~cm}^{-1}$ responsible for the presence of the carbonyl group. This bond is formed during the degradation process of the material during ageing. UV ageing causes the largest change in the color of the elastomer, which may result from the simultaneous action of many degradants, which are moisture, UV rays and high temperature.

Understanding the degradation processes of polymer matrix under the influence of various external factors and predicting the lifetime of polymeric materials is very important for better design of processes, manufacturing and for industry.

This study was supported by the National Centre for Research and Development (NCBR) project: LIDER/32/0139/L-7/15/NCBR/2016.

\section{References}

1. L. W. McKeen, Permeability Properties of Plastics and Elastomers, 178 (2011)

2. L.W. McKeen, L. K. Massey, Film Properties of Plastics and Elastomers, 2nd Edition, 125 (2004) 
3. C. R. Blass, Polymers in Disposable Medical Devices: A European Perspective, 43 (1999)

4. Y.N. Vodyanitskii, N.P. Kirillova, Moscow Univ. Soil Sci. Bull. 71, 139 (2016) 